Supporting Information for

\title{
Molecular Origin of Ultrafast Water-Protein Coupled Interactions
}

\author{
Yangzhong Qin, ${ }^{1,2,}$ Menghui Jia, ${ }^{1, \pi}$ Jin Yang, ${ }^{2}$ Dihao Wang, ${ }^{2}$ Lijuan Wang, ${ }^{2}$ \\ Jianhua $\mathrm{Xu}^{1, *}$ and Dongping Zhong ${ }^{2, *}$
}

${ }^{1}$ State Key Laboratory of Precision Spectroscopy, East China Normal University, Shanghai 200062, China. ${ }^{2}$ Department of Physics, Department of Chemistry and Biochemistry, and Programs of Biophysics, Chemical Physics and Biochemistry, The Ohio State University, Columbus, Ohio 43210, USA

*Corresponding author. E-mail: jhxu@phy.ecnu.edu.cn or zhong.28@osu.edu. 


\section{Material and Methods}

\section{Sample Temperature Control and Calibration}

We used a home-made "spin cell" to hold the sample. The sample was sandwiched by two round quartz windows ( $1 \mathrm{~cm}$ diameter, $2 \mathrm{~mm}$ thick) with a $1 \mathrm{~mm}$ thick Teflon spacer to fill the sample in the middle. The sample quartz cell was further sandwiched by two metal plates to form a complete spin cell. The spin cell was then inserted into an aluminum block holder, which is connected to a water bath (VWR scientific water bath). Before we started the ultrafast fluorescence measurement, we always carefully measured the sample temperature. A very thin thermocouple (wire has a 0.0799-mm diameter) was directly inserted into the sample space with SNase wild type solution. Note, both thermometer (HH92) and thermocouple (5SC-TT-K-40-36) were purchased from Omega Engineering, INC. They were calibrated according to the manufacture manual after we received them and further tested the accuracy from $0{ }^{\circ} \mathrm{C}$ to $70^{\circ} \mathrm{C}$. The accuracy was determined to be $\pm 0.3{ }^{\circ} \mathrm{C}$. We also measured the temperature at different spots of the sample from the side to the center. The difference is within $\pm 0.4^{\circ} \mathrm{C}$ at most. While we were measuring the sample temperature, we also measured the temperature of the aluminum block (spin cell holder). Such temperature values were recorded and paired with the directly measured sample temperatures to form a table that can be checked later to refer the sample temperature. We constantly monitored the temperature of the aluminum block during the laser experiment to ensure that the sample temperature does not change due to environment fluctuation (the temperature in our laser lab fluctuates at most $\pm 0.5^{\circ} \mathrm{C}$ ).

\section{Fluorescence Anisotropy Measurement}

The fluorescence anisotropy measurement has to be done carefully. Otherwise, it is easy to miss the first intermediate dynamics $(\sim 10 \mathrm{ps})$ during the experiment (see Supplementary Figure 1). The reason is that the anisotropy experiment requires a longtime average of the fluorescence signal at both parallel and perpendicular polarizations. But typically, most protein samples cannot withstand longtime laser illumination and the fluorescence signal would show different. To avoid this problem, we take two major steps to avoid this problem: one is change to fresh protein sample before it is damaged. We check the transient every 50 scans to make sure that the transient dynamics do not change with time. We found that our protein sample is stable with 200 scans. After 200 scans it starts to exhibit different dynamics. Therefore, we changed the protein sample before 200 scans. The other way is to reduce the pump pulse energy to 100 $\mathrm{nJ} /$ pulse. Note that this pump energy is very weak comparing to those often used in many chemistry experiments (on the order of $\sim \mu \mathrm{J} /$ pulse.) Another key point is that both parallel and perpendicular transients should be taken under the same condition, especially with the same time. Typically, the perpendicular signal is weaker than the parallel signal, but one should not shoot the protein sample for longer time to achieve a comparable signal-to-noise ratio to the parallel signal without changing to the fresh sample.

\section{Data Analysis}

The methodology of detailed fluorescence spectroscopic data analysis was reported elsewhere $^{1,2}$. Briefly, we measured the solvation dynamics by monitoring the dynamic Stokes shift and defining the solvation correlation function as the following: 


$$
C(t)=\frac{v_{s}(t)-v_{l}(t)}{v_{s}(0)-v_{l}(0)}
$$

where $v_{\mathrm{s}}$ is the overall emission peak including solvation dynamics and population decay contributions. $v_{1}$ is the total lifetime emission peak. Typically, the fluorescence transients at certain wavelength $I_{\lambda}(t)$ can be well fitted with multiple exponential decays after deconvolution with instrument response function (IRF). Therefore, we can separate the contributions from solvation and lifetimes by defining the following formula:

$$
I_{\lambda}(t)=I_{\lambda}^{\text {solv }}(t)+I_{\lambda}^{\text {popul }}(t)=\sum_{i} a_{i} e^{-t / \tau_{i}}+\sum_{j} b_{j} e^{-t / \tau_{j}}
$$

the first term is for the solvation and the second term is for the lifetime emission. The coefficient $\mathrm{a}_{\mathrm{i}}$ is positive (decay dynamics) at the blue side of the emission peak and is negative (initial rise) at the red side. The coefficient $b_{j}$ is always positive and represents relative contributions of multiple lifetime decays. With the steady-state relative emission intensity $I_{\lambda}^{s s}$ at $\lambda$, we can easily construct the overall emission spectrum from eq. (S3) and the lifetime emission spectrum from eq. (S4) below.

$$
\begin{gathered}
I(\lambda, t)=\frac{I_{\lambda}^{s S} I_{\lambda}(t)}{\sum_{i} a_{i} \tau_{i}+\sum_{j} b_{j} \tau_{j}} \\
I^{\text {popul }}(\lambda, t)=\frac{I_{\lambda}^{s S} I_{\lambda}^{\text {popul }}(t)}{\sum_{i} a_{i} \tau_{i}+\sum_{j} b_{j} \tau_{j}}
\end{gathered}
$$

Therefore, we can construct the solvation correlation function by following eq. (S1). Same as solvation components in eq. (S2), the solvation correlation function can be fitted with multiple exponential decay indicating different hydration water motions.

The fluorescence anisotropy directly measures the tryptophan rotational dynamics. With the two femtosecond-resolved fluorescence intensity of $I_{/ /}$and $I_{\perp}$, we can construct the fluorescence anisotropy by following eq. (S5)

$$
r(t)=\frac{I_{/ /}(t)-I_{\perp}(t)}{I_{/ /}(t)+2 I_{\perp}(t)}
$$

The anisotropy typically shows multiple decay dynamics which correlate to two different molecular mechanisms. Therefore, we can write it in another form (S6),

$$
r(t)=r_{I C}(t)+r_{2 W}(t)+r_{3 W}(t)+r_{T}(t)
$$

The initial ultrafast decay $\left(r_{I C}\right)$ results from the internal conversion between two electronically excited states of ${ }^{1} \mathrm{~L}_{\mathrm{a}}$ and ${ }^{1} \mathrm{~L}_{\mathrm{b}}$ happening in less than $100 \mathrm{fs} .{ }^{3}$ The following two components $\left(r_{2 W}\right.$ and $r_{3 W}$ ) indicate the local wobbling motion of the tryptophan sidechain. $r_{T}$ represents the contribution from the overall protein tumbling motion. Following a simple axially symmetric oscillation model, ${ }^{4}$ we can estimate the wobbling semiangle $(\theta)$ using the following expression: 


$$
1-\frac{r_{i W}(0)}{r_{i W}(0)+r_{T}(0)}=\left[\frac{3 \cos ^{2} \theta-1}{2}\right]^{2}, \mathrm{i}=2,3
$$

Each fluorescence anisotropy component from wobbling and tumbling motions follows an exponential decay and their decay timed can be determined from exponential fits.

\section{MD simulation}

The amber package 11 was used for the MD simulation with ff99SB force field. The crystal structure was taken from the protein data bank (1SNO). Mutation was performed in the Pymol software and chosen the most probable configuration as the initial structure. Then the whole protein was solvated by explicit water TIP3P with a cutoff of $12 \AA$ in an octahedral box. $\mathrm{Cl}^{-1}$ and $\mathrm{Na}^{+}$ions were added to neutralize the system. A minimization process was used to remove the bad contacts. First, fix the solute and run 1000 steps of minimization to relax the water and ion molecules with the constant volume periodic boundaries; second, relax the entire system without any constraints for 1500 steps. And then, while adding a weak restraint on the protein molecules, the system was heated up from 0 to $300 \mathrm{~K}$ in 10000 steps with a step size of 2 fs by using the Langevin dynamics to control temperature. SHAKE algorithm was used to constrain bonds involving hydrogen. Finally, a $20 \mathrm{~ns}$ MD simulation trajectory was obtained after a 0.5-ns equilibration process at $300 \mathrm{~K}$ and under the constant pressure of 1 atm to mimic the typical experiment condition. 


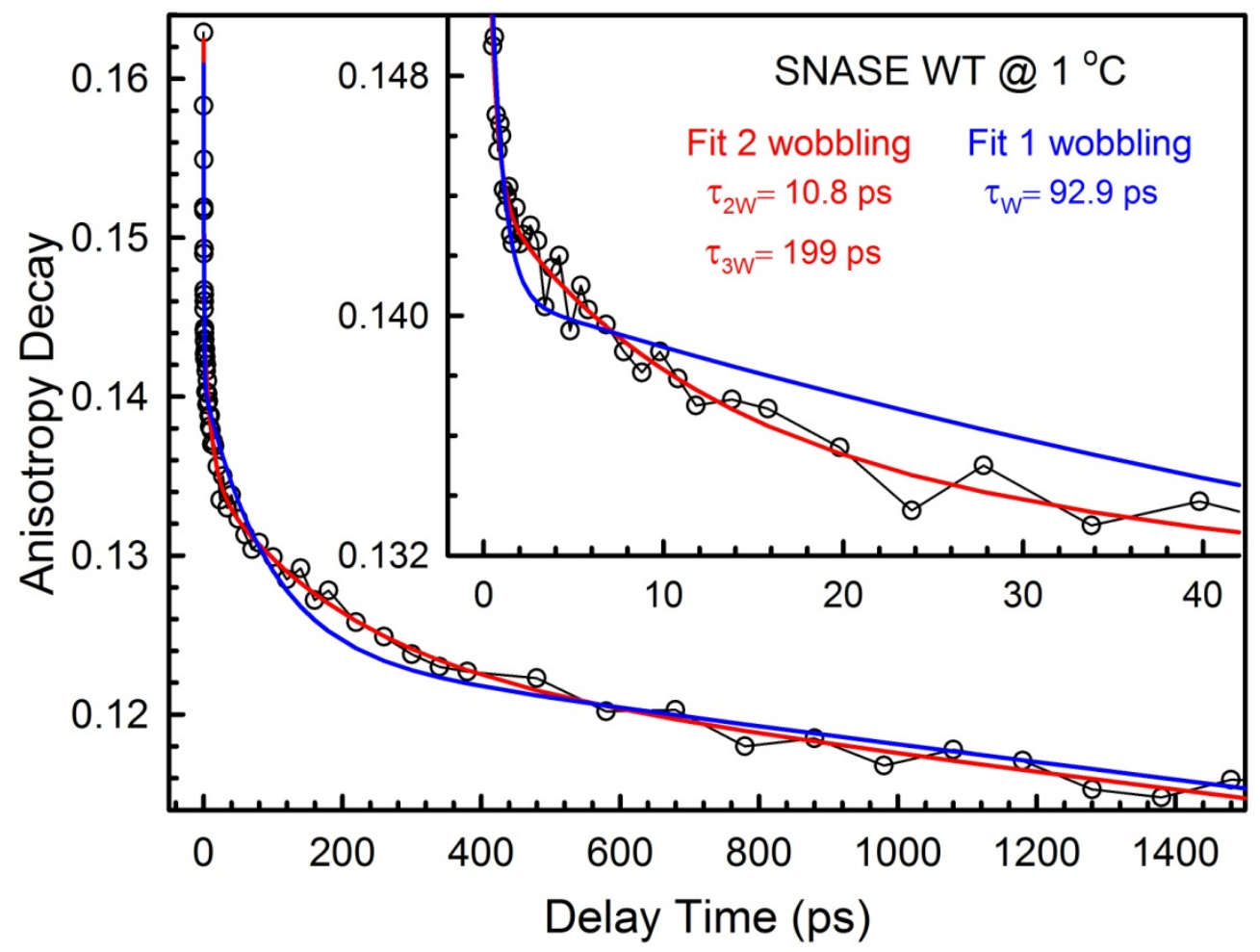

Figure S1. Fluorescence anisotropy decay of SNase WT at $1{ }^{\circ} \mathrm{C}$. The scatter plots show the measured fluorescence anisotropy and the solid lines show two different fittings. Red line indicates the fitting with two exponential components for the wobbling besides initial ultrafast decay $(<0.5 \mathrm{ps})$ and last slow tumbling decay in nanosecond. Blue line indicates the fitting with only one exponential component for the wobbling. The inset shows the anisotropy decay in a short time window. 


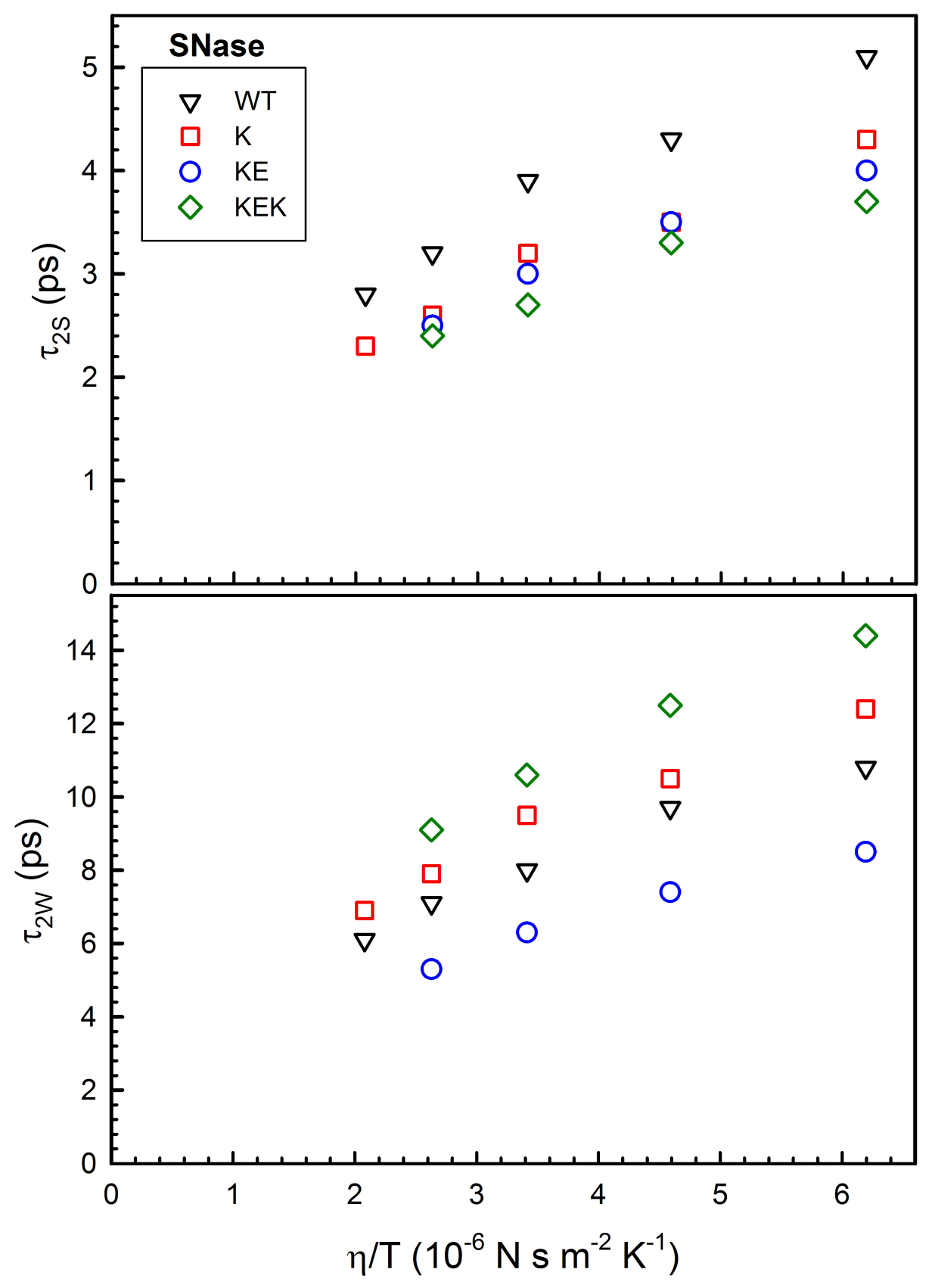

Figure S2. Variation of the relaxation time $\left(\tau_{2 S}\right.$ and $\left.\tau_{2 \mathrm{~W}}\right)$ with $\eta / T . \eta$ is the dynamic viscosity of water and $\mathrm{T}$ is the absolute temperature in Kelvin. The scatter plots are the measured relaxation times, which do not follow either Debye model or Perrin's relation., 


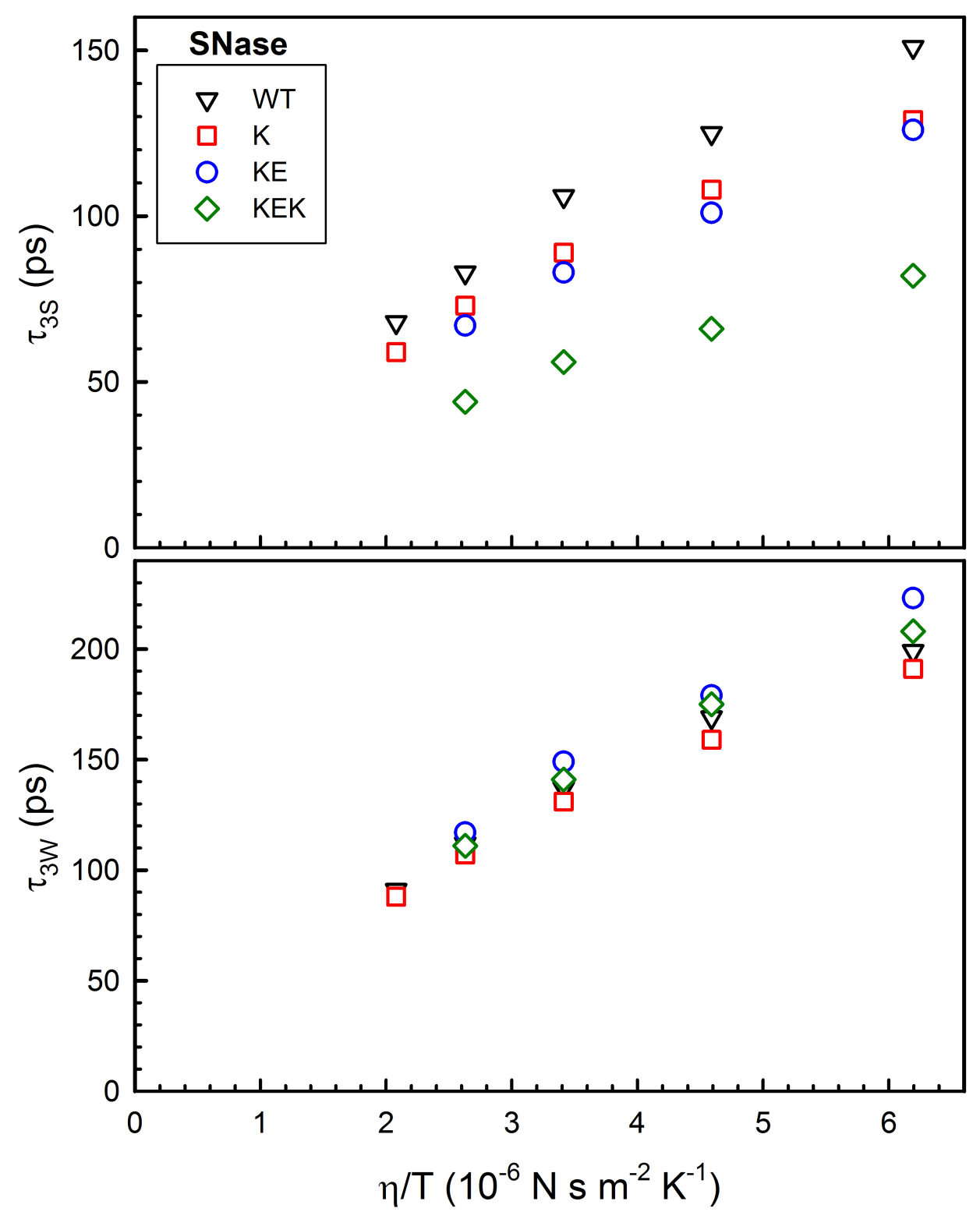

Figure S3. Variation of the relaxation time ( $\tau_{3 \mathrm{~S}}$ and $\tau_{3 \mathrm{w}}$ ) with $\eta / T$. $\eta$ is the dynamic viscosity of water and $\mathrm{T}$ is the absolute temperature in Kelvin. The scatter plots are the measured relaxation times, which do not follow either Debye model or Perrin's relation. ${ }^{5,6}$ 


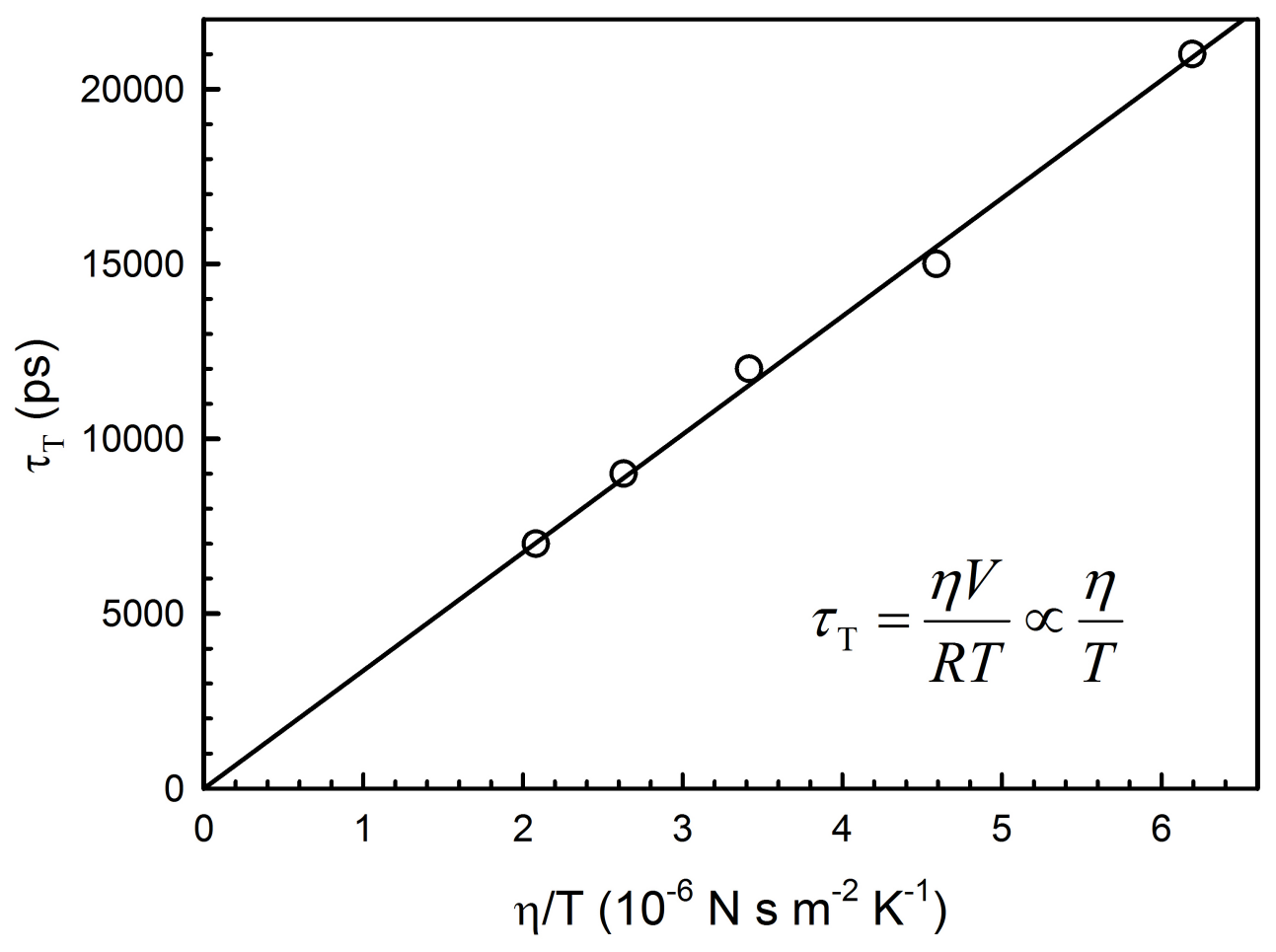

Figure S4. Variation of the tumbling time $\left(\tau_{\mathrm{T}}\right)$ with $\eta / T . \eta$ is the dynamic viscosity of pure water and $\mathrm{T}$ is the absolute temperature in Kelvin. The tumbling time of the whole protein molecules obeys the Perrin's relation $\tau_{T}=\frac{\eta V}{R T}$, ${ }^{6}$ where $\mathrm{V}$ is the molecular volume and $\mathrm{R}$ is the gas constant. 
Table S1. Fitting results for solvation dynamics and tryptophan anisotropy dynamics.

\begin{tabular}{|c|c|c|c|c|c|c|c|c|c|}
\hline \multicolumn{10}{|c|}{ Wild Type } \\
\hline Temperature $\left({ }^{\circ} \mathrm{C}\right)$ & $\begin{array}{l}\text { A2 } \\
(\%) \\
\end{array}$ & $\begin{array}{l}\text { A3 } \\
(\%) \\
\end{array}$ & $\tau_{2 \mathrm{~S}}(\mathrm{ps})$ & $\begin{array}{c}\tau_{3 \mathrm{~S}} \\
(\mathrm{ps}) \\
\end{array}$ & $\begin{array}{r}\mathrm{E}_{\mathrm{Tot}} \\
\left(\mathrm{cm}^{-1}\right)\end{array}$ & $\begin{array}{c}\mathrm{E}_{2} \\
\left(\mathrm{~cm}^{-1}\right) \\
\end{array}$ & $\begin{array}{c}\mathrm{E}_{3} \\
\left(\mathrm{~cm}^{-1}\right) \\
\end{array}$ & $\begin{array}{l}\tau_{2 \mathrm{~W}} \\
(\mathrm{ps}) \\
\end{array}$ & $\begin{array}{l}\tau_{3 \mathrm{~W}} \\
(\mathrm{ps}) \\
\end{array}$ \\
\hline 1 & 65.2 & 34.8 & 5.1 & 151 & 747 & 487 & 260 & 10.8 & 199 \\
\hline 10 & 66.2 & 33.8 & 4.3 & 125 & 753 & 499 & 254 & 9.7 & 169 \\
\hline 20 & 64.8 & 35.2 & 3.9 & 106 & 759 & 492 & 267 & 8.0 & 137 \\
\hline 30 & 65.1 & 34.9 & 3.2 & 83 & 814 & 530 & 284 & 7.1 & 112 \\
\hline 40 & 66.6 & 33.4 & 2.8 & 68 & 840 & 559 & 281 & 6.1 & 91 \\
\hline \multicolumn{10}{|c|}{$\mathrm{K}(\mathrm{K} 110 \mathrm{~A})$} \\
\hline Temperature $\left({ }^{\circ} \mathrm{C}\right)$ & $\begin{array}{l}\text { A2 } \\
(\%)\end{array}$ & $\begin{array}{l}\text { A3 } \\
(\%)\end{array}$ & $\tau_{2 \mathrm{~S}}(\mathrm{ps})$ & $\begin{array}{c}\tau_{3 \mathrm{~S}} \\
(\mathrm{ps}) \\
\end{array}$ & $\begin{array}{c}\mathrm{E}_{\mathrm{Tot}} \\
\left(\mathrm{cm}^{-1}\right)\end{array}$ & $\begin{array}{c}\mathrm{E}_{2} \\
\left(\mathrm{~cm}^{-1}\right) \\
\end{array}$ & $\begin{array}{c}\mathrm{E}_{3} \\
\left(\mathrm{~cm}^{-1}\right) \\
\end{array}$ & $\begin{array}{l}\tau_{2 \mathrm{~W}} \\
(\mathrm{ps}) \\
\end{array}$ & $\begin{array}{l}\tau_{3 \mathrm{~W}} \\
(\mathrm{ps}) \\
\end{array}$ \\
\hline 1 & 62.0 & 38.0 & 4.3 & 129 & 799 & 496 & 303 & 12.4 & 191 \\
\hline 10 & 64.8 & 35.2 & 3.5 & 108 & 804 & 521 & 283 & 10.5 & 159 \\
\hline 20 & 65.5 & 34.5 & 3.2 & 89 & 837 & 548 & 289 & 9.5 & 131 \\
\hline 30 & 65.5 & 34.5 & 2.6 & 73 & 820 & 537 & 283 & 7.9 & 107 \\
\hline 40 & 67.2 & 32.8 & 2.3 & 59 & 860 & 578 & 282 & 6.9 & 88 \\
\hline \multicolumn{10}{|c|}{ KE (K110A E129A) } \\
\hline Temperature $\left({ }^{\circ} \mathrm{C}\right)$ & $\begin{array}{l}\text { A2 } \\
(\%)\end{array}$ & $\begin{array}{l}\text { A3 } \\
(\%)\end{array}$ & $\tau_{2 \mathrm{~S}}(\mathrm{ps})$ & $\begin{array}{c}\tau_{3 \mathrm{~S}} \\
(\mathrm{ps})\end{array}$ & $\begin{array}{c}\mathrm{E}_{\mathrm{Tot}} \\
\left(\mathrm{cm}^{-1}\right)\end{array}$ & $\begin{array}{c}\mathrm{E}_{2} \\
\left(\mathrm{~cm}^{-1}\right)\end{array}$ & $\begin{array}{c}\mathrm{E}_{3} \\
\left(\mathrm{~cm}^{-1}\right) \\
\end{array}$ & $\begin{array}{l}\tau_{2 \mathrm{~W}} \\
(\mathrm{ps}) \\
\end{array}$ & $\begin{array}{l}\tau_{3 \mathrm{~W}} \\
(\mathrm{ps}) \\
\end{array}$ \\
\hline 1 & 69.5 & 30.5 & 4.0 & 126 & 807 & 561 & 246 & 8.5 & 223 \\
\hline 10 & 70.2 & 29.8 & 3.5 & 101 & 857 & 602 & 255 & 7.4 & 179 \\
\hline 20 & 70.4 & 29.6 & 3.0 & 83 & 884 & 623 & 261 & 6.3 & 149 \\
\hline 30 & 68.9 & 31.1 & 2.5 & 67 & 895 & 617 & 278 & 5.3 & 117 \\
\hline \multicolumn{10}{|c|}{ KEK (K110A E129A K133A) } \\
\hline Temperature $\left({ }^{\circ} \mathrm{C}\right)$ & $\begin{array}{l}\text { A2 } \\
(\%) \\
\end{array}$ & $\begin{array}{l}\text { A3 } \\
(\%) \\
\end{array}$ & $\tau_{2 S}(\mathrm{ps})$ & $\begin{array}{c}\tau_{3 \mathrm{~S}} \\
(\mathrm{ps}) \\
\end{array}$ & $\begin{array}{c}\mathrm{E}_{\mathrm{Tot}} \\
\left(\mathrm{cm}^{-1}\right)\end{array}$ & $\begin{array}{c}\mathrm{E}_{2} \\
\left(\mathrm{~cm}^{-1}\right) \\
\end{array}$ & $\begin{array}{c}\mathrm{E}_{3} \\
\left(\mathrm{~cm}^{-1}\right) \\
\end{array}$ & $\begin{array}{l}\tau_{2 \mathrm{~W}} \\
(\mathrm{ps}) \\
\end{array}$ & $\begin{array}{l}\tau_{3 \mathrm{~W}} \\
(\mathrm{ps}) \\
\end{array}$ \\
\hline 1 & 72.5 & 27.5 & 3.7 & 82 & 838 & 607 & 231 & 14.4 & 208 \\
\hline 10 & 70.6 & 29.4 & 3.3 & 66 & 882 & 622 & 260 & 12.5 & 175 \\
\hline 20 & 72.7 & 27.3 & 2.7 & 56 & 1017 & 740 & 277 & 10.6 & 141 \\
\hline 30 & 68.1 & 31.9 & 2.4 & 44 & 1074 & 731 & 343 & 9.1 & 111 \\
\hline
\end{tabular}




\section{References}

(1) Zhang, L.; Yang, Y.; Kao, Y.; Wang, L.; Zhong, D. Protein Hydration Dynamics and Molecular Mechanism of Coupled Water-Protein Fluctuations. J. Am. Chem. Soc. 2009, 131, 10677-10691.

(2) Qin, Y.; Chang, C.-W.; Wang, L.; Zhong, D. Validation of Response Function Construction and Probing Heterogeneous Protein Hydration by Intrinsic Tryptophan. J. Phys. Chem. B 2012, 116, 13320-13330.

(3) Yang, J.; Zhang, L.; Wang, L.; Zhong, D. Femtosecond Conical Intersection Dynamics of Tryptophan in Proteins and Validation of Slowdown of Hydration Layer Dynamics. J. Am. Chem. Soc. 2012, 134, 16460-16463.

(4) Steiner, R. F. In Topics in Fluorescence Spectroscopy; Lakowicz, J. R., Ed.; Plenum: New York, 1991; Vol.2, pp 1-51.

(5) Agmon, N. Tetrahedral Displacement: The Molecular Mechanism behind the Debye Relaxation in Water. J. Phys. Chem. 1996, 100, 1072-1080.

(6) Principles of Fluorescence Spectroscopy, Lakowicz, J. R., Ed.; Springer: New York, 2006.

\section{Complete reference (6)}

(6) Schiro, G.; Fichou, Y.; Gallat, F. X.; Wood, K.; Gabel, F.; Moulin, M.; Hartlein, M.; Heyden, M.; Colletier, J. P.; Orecchini, A.; Paciaroni, A.; Wuttke, J.; Tobias, D. J.; Weik, M. Translational Diffusion of Hydration Water Correlates with Functional Motions in Folded and Intrinsically Disordered Proteins. Nat. Commun. 2015, 6, 6490. 